\title{
Video-assisted thoracic surgery versus open lobectomy for lung cancer: A secondary analysis of data from the American College of Surgeons Oncology Group Z0030 randomized clinical trial
}

\author{
Walter J. Scott, MD, FACS, ${ }^{a}$ Mark S. Allen, MD, ${ }^{\mathrm{b}}$ Gail Darling, MD, FRCSC, FACS, ${ }^{\mathrm{c}}$ Bryan Meyers, MD, ${ }^{\mathrm{d}}$ \\ Paul A. Decker, MS, ${ }^{\mathrm{b}}$ Joe B. Putnam, MD, ${ }^{\mathrm{e}}$ Robert W. Mckenna, MD, ${ }^{\mathrm{f}}$ Rodney J. Landrenau, MD, ${ }^{\mathrm{g}}$ \\ David R. Jones, MD, ${ }^{\mathrm{h}}$ Richard I. Inculet, MD, ${ }^{\mathrm{i}}$ and Richard A. Malthaner, $\mathrm{MD}^{\mathrm{i}}$
}

\begin{abstract}
Objective: Video-assisted thoracoscopic lobectomy remains controversial. We compared outcomes from participants in a randomized study comparing lymph node sampling versus dissection for early-stage lung cancer who underwent either video-assisted thoracoscopic or open lobectomy.
\end{abstract}

\begin{abstract}
Methods: Data from 964 participants in the American College of Surgeons Oncology Group Z0030 trial were used to construct propensity scores for video-assisted thoracoscopic versus open lobectomy (based on age, gender, histology, performance status, tumor location, and T1 vs T2). Propensity scores were used to estimate the adjusted risks of short-term outcomes of surgery. Patients were classified into 5 equal-sized groups and compared using conditional logistic regression or repeated measures analysis of variance.
\end{abstract}

\begin{abstract}
Results: A total of 752 patients (66 video-assisted and 686 open procedures) were analyzed on the basis of propensity score stratification. Median operative time was shorter for video-assisted thoracoscopic lobectomy (video-assisted thoracoscopy 117.5 minutes vs open 171.5 minutes; $P<.001$ ). Median total number of lymph nodes retrieved (dissection group only) was similar (video-assisted thoracoscopy 15 nodes vs open 19 nodes; $P=.147$ ), as were instances of $\mathrm{R} 1 / \mathrm{R} 2$ resection (video-assisted thoracoscopy $0 \%$ vs open $2.3 \% ; P=.368$ ). Patients undergoing video-assisted thoracoscopic lobectomy had less atelectasis requiring bronchoscopy $(0 \%$ vs $6.3 \%, P=.035)$, fewer chest tubes draining greater than 7 days $(1.5 \%$ vs $10.8 \% ; P=.029)$, and shorter median length of stay ( 5 days vs 7 days; $P<.001$ ). Operative mortality was similar (video-assisted thoracoscopy $0 \%$ vs open $1.6 \%, P=1.0)$.
\end{abstract}

Conclusion: Patients undergoing video-assisted lobectomy had fewer respiratory complications and shorter length of stay. These data suggest video-assisted thoracoscopic lobectomy is safe in patients with resectable lung cancer. Longer follow-up is needed to determine the oncologic equivalency of video-assisted versus open lobectomy. (J Thorac Cardiovasc Surg 2010;139:976-83)

The potential benefits of video-assisted thoracoscopic (VATS) lobectomy include faster patient recovery, fewer complications, and shorter hospital stay without compromising the oncologic aspects of the operation. However, the routine use of VATS lobectomy for the treatment of resectable non-small cell lung cancer (NSCLC) remains controversial. Data supporting the use of VATS lobectomy are from

\footnotetext{
From the Fox Chase Cancer Center, ${ }^{\text {a }}$ Philadelphia, Pa; Mayo Clinic, ${ }^{\text {b }}$ Rochester, Minn; University of Toronto, ${ }^{\mathrm{c}}$ Toronto, Canada; Washington University of St Louis, ${ }^{\mathrm{d}} \mathrm{St}$ Louis, Mo; Vanderbilt, University Medical Center, ${ }^{\mathrm{e}}$ Nashville, Tenn; University of California, ${ }^{\mathrm{f}}$ Los Angeles, Calif; Allegheny General Hospital, ${ }^{\mathrm{g}}$ Pittsburgh, Pa; University of Virginia, ${ }^{\mathrm{h}}$ Charlottesville, Va; and London Health Science Center, ${ }^{\mathrm{i}}$ London, Ontario, Canada.

Disclosures: None.

Supported by National Cancer Institute U10 grant CA076001.

Received for publication June 19, 2009; revisions received Sept 30, 2009; accepted for publication Nov 22, 2009; available ahead of print Feb 22, 2010.

Address for reprints: Walter J. Scott, MD, FACS, Fox Chase Cancer Center, Department of Surgical Oncology, 7701 Burholme Avenue, Philadelphia, PA 19111 (E-mail: walter.scott@fccc.edu).

0022-5223/\$36.00

Copyright (c) 2010 by The American Association for Thoracic Surgery doi: $10.1016 /$ j.jtcvs.2009.11.059
}

randomized trials, ${ }^{1-4}$ a multicenter phase II study, ${ }^{5}$ case-control series, ${ }^{6-9}$ and a large retrospective series. ${ }^{10}$ Two recent meta-analyses have been published. ${ }^{6,7}$ The randomized trials enrolled relatively small numbers of patients, and retrospective case series are subject to selection biases. Recent publications have been case-control series. ${ }^{8,9}$

The American College of Surgeons Oncology Group (ACOSOG) Z0030 clinical trial provided an opportunity to retrospectively compare VATS lobectomy with open lobectomy. ${ }^{11} \mathrm{Z} 0030$ is a randomized, prospective, multi-institutional clinical trial that was designed to determine the effect on survival of lymph node sampling versus mediastinal lymph node dissection in patients undergoing complete resection of early-stage NSCLC. A secondary objective was to determine the relative morbidity of the 2 techniques. Although the final study end points of survival have not been reported, data on short-term outcomes after lung resection are available. ${ }^{11}$ We used propensity score-based matching $^{12}$ to compare the short-term clinical outcomes of 2 groups of patients who enrolled in the trial: those who underwent VATS lobectomy and those who underwent open lobectomy. 


\section{Abbreviations and Acronyms \\ ACOSOG $=$ American College of Surgeons Oncology Group \\ $\mathrm{CI}=$ confidence interval \\ NSCLC $=$ non-small cell lung cancer \\ $\mathrm{RR} \quad=$ relative risk \\ VATS $=$ video-assisted thoracoscopic}

\section{PATIENTS AND METHODS}

The details of the study design, eligibility requirements, and morbidity and mortality for patients enrolled in the ACOSOG Z0030 trial have been reported. In summary, ${ }^{11}$ eligible patients were required to be 18 years of age or older, to have an Eastern Cooperative Oncology Group performance score less than 3, and to have a tissue diagnosis of a clinically resectable T1 or T2, N0 or non-hilar N1, M0 NSCLC (squamous cell carcinoma, large cell carcinoma, or adenocarcinoma, including bronchoalveolar carcinoma) established before randomization. Patients underwent computed tomography of the chest and upper abdomen, including the liver and adrenal glands within 60 days of the pulmonary resection. Patients who had a pre-thoracotomy mediastinoscopy were eligible if no mediastinal lymph node metastases were identified. Patients who did not undergo mediastinoscopy were eligible if they had no evidence of mediastinal lymphadenopathy by computed tomography criteria $(>1.0 \mathrm{~cm}$ in the shortest axis). Eligible patients had to be candidates for a complete resection of the carcinoma by means of pneumonectomy, lobectomy, bilobectomy, or anatomic segmentectomy, with or without sleeve resection. The type of resection (VATS or open) was not specified in the protocol. Exclusion criteria included patients with T3 or $\mathrm{T} 4$ tumors, patients who were treated with pulmonary wedge excision, and patients who received prior chemotherapy or radiation therapy for their cancer. Additional intraoperative eligibility criteria included pre-randomization sampling of designated lymph node stations. Patients with N2 metastases on frozen section were excluded from randomization. Therefore, all patients in ACOSOG Z0030 had as a minimum either mediastinoscopy and/or intraoperative lymph node sampling for nodal staging.

All surgeons who participated in the trial were general thoracic surgeons. The enrolling surgeons were required to read a description of the procedure and watch a video of the mediastinal lymph node dissection technique before enrolling their first patient into the trial. Each operative note was reviewed by the principle investigators for completeness of the dissection and adherence to the protocol. All patients provided written informed consent before trial enrollment in accordance with applicable guidelines. The ACOSOG Z0030 trial was approved by a local Human Investigations Committee and in accord with an assurance filed with and approved by the Department of Health and Human Services.

We reviewed the ACOSOG Z0030 data set and identified 964 patients (66 with VATS and 898 with open lobectomy) who underwent lobectomy, bilobectomy, or anatomic segmentectomy. Those who underwent pneumonectomy or wedge resection alone were excluded from this analysis. Patients who underwent VATS wedge resection for diagnosis and then open thoracotomy and resection as described above were included in the "open lobectomy" group.

Differences between groups in clinical and tumor characteristics were compared using the 2-sample rank test or chi-square test as appropriate. Clinical and tumor characteristics were used to build a propensity score for choice of treatments. ${ }^{12-14}$ These variables included age, gender, histology, performance status, tumor location, and clinical stage (T1 vs T2). Propensity scores were developed to estimate the adjusted risks of perioperative outcomes associated with the choice of treatment (VATS vs open). Logistic regression was used to estimate the probability of VATS versus open given the previously listed risk factors. Patients were classified into 7 groups on the basis of their propensity scores. A total of 208 patients undergoing thoracotomy had lower scores than the lowest score of any patient treated with VATS (group 0); 4 patients undergoing open lobectomy had higher scores than the highest score of any patient treated with VATS (group 6). Patients from these 2 groups were omitted from further analysis. The remaining 752 patients (66 in the VATS group and 686 in the open lobectomy group) were classified into 5 equal-sized propensity score groups (groups 1-5). Conditional logistic regression with 5 strata (propensity score groups $1-5$ ) for dichotomous outcomes and repeated-measures analysis of variance with strata as a repeated factor for continuous outcomes were used to compare outcomes between VATS and open cases.

The 66 patients in the VATS group were older than the 686 patients in the open group, with a median age of 72.9 years (mean \pm standard deviation, $70.9 \pm 9.7$ ) compared with 68.6 years (mean \pm standard deviation, 68.1 $\pm 8.8 ; P=.011$ ) (Table 1). Despite the age difference, there was a higher percentage of patients with performance status 0 in the VATS group compared with the open group $(90.9 \%$ vs $71.1 \% ; P=.002)$. There was also a difference in histology with a higher percentage of adenocarcinoma in the VATS group compared with the open group. In the VATS cohort, 44 patients $(66.7 \%)$ presented with $\mathrm{T} 1$ tumors and 22 patients $(33.3 \%)$ presented with $\mathrm{T} 2$ tumors. In the open cohort, 408 patients $(59.5 \%)$ presented with T1 tumors and 278 patients $(40.5 \%)$ presented with T2 tumors $(P=$ .255). The distribution of tumor location was similar for each group with most tumors in the upper lobes of the lung (Table 1). Because the variables of age, gender, histology, performance status, tumor location, and clinical stage ( $\mathrm{T} 1 \mathrm{vs} \mathrm{T} 2$ ) were included in the propensity score model, the differences between the groups demonstrated in Table 1 were adjusted for in the statistical analysis.

The VATS group consisted of 66 patients from 8 institutions, and the open lobectomy group consisted of 686 patients from 71 institutions. The highest accruing surgeon to the VATS lobectomy group contributed 54 of 66 patients $(82 \%)$.

\section{RESULTS}

Perioperative outcomes are reported in Table 2. Operative mortality was similar for the 2 cohorts. Median operative time was shorter for the VATS cohort compared with the open group. The occurrence of bleeding requiring transfusion and the number of instances of bleeding requiring reoperation were each similar for the 2 cohorts. The median amount of chest tube drainage was less for the VATS group, as was the duration of chest tube drainage. Median hospital length of stay was also less for the VATS group compared with the open group ( 5 vs 7 days; $P<.001$ ). The incidence of microscopic (R1) or grossly positive (R2) resection margins was similar for each group. Because this is a post hoc analysis of data from a trial that randomized patients between lymph node sampling and dissection, we specifically analyzed those patients who were randomized to receive lymph node dissection. The median number of lymph node stations sampled was similar for the 2 cohorts, as was the median number of lymph nodes that were removed from each group.

A list of complications for both groups is provided in Table 3. Instances of chest tube drainage lasting more than 7 days were noted less often in the VATS group compared with the open group, whereas the occurrence of air leak lasting 
TABLE 1. Characteristics of subjects in each group

\begin{tabular}{|c|c|c|c|}
\hline Variable & $\operatorname{VATS}(n=66)$ & Open $(n=686)$ & $P^{*}$ \\
\hline Age, y & $72.9 ; 70.9 \pm 9.7$ & $68.6 ; 68.1 \pm 8.8$ & .011 \\
\hline Gender & & & .148 \\
\hline Female & $38(57.6)$ & $331(48.3)$ & \\
\hline Male & $28(42.4)$ & $355(51.8)$ & \\
\hline Histology & & & .029 \\
\hline Squamous & $10(15.2)$ & $206(30.0)$ & \\
\hline Adenocarcinoma & $45(68.2)$ & $354(51.6)$ & \\
\hline Large cell & $2(3.0)$ & $36(5.3)$ & \\
\hline Bronchoalveolar & $8(12.1)$ & $57(8.3)$ & \\
\hline Other non-small cell & $1(1.5)$ & $33(4.8)$ & \\
\hline ECOG performance status & & & .002 \\
\hline 0 & $60(90.9)$ & $488(71.1)$ & \\
\hline 1 & $5(7.6)$ & $192(28.0)$ & \\
\hline 2 & $1(1.5)$ & $6(0.9)$ & \\
\hline \multicolumn{4}{|l|}{ Tumor location $\dagger$} \\
\hline RUL & $32(48.5)$ & $284(41.4)$ & .265 \\
\hline RML & $2(3.0)$ & $44(6.4)$ & .273 \\
\hline RLL & $8(12.1)$ & $112(16.3)$ & .373 \\
\hline LUL & $18(27.3)$ & $173(25.2)$ & .714 \\
\hline LLL & $7(10.6)$ & $85(12.4)$ & .673 \\
\hline Clinical stage & & & .255 \\
\hline $\mathrm{T} 1$ & $44(66.7)$ & $408(59.5)$ & \\
\hline $\mathrm{T} 2$ & $22(33.3)$ & $278(40.5)$ & \\
\hline \multicolumn{4}{|l|}{ Procedure performed } \\
\hline Lobectomy & $63(95.5)$ & $606(88.3)$ & .078 \\
\hline Bilobectomy & $1(1.5)$ & $27(3.9)$ & .502 \\
\hline Segmentectomy & $3(4.6)$ & $54(7.9)$ & .330 \\
\hline
\end{tabular}

$E C O G$, Eastern Cooperative Oncology Group; $R U L$, right upper lobe; $R M L$, right middle lobe; $R L L$, right left lobe; $L U L$, left upper lobe; $L L L$, left lower lobe. Values are $\mathrm{n}$ $(\%)$ or median; mean \pm standard deviation. $*$ Chi-square test for categoric variables and 2-sample rank sum test for continuous variables. †ेMultiple tumor locations.

greater than 7 days was similar for the 2 groups. Adult respiratory distress syndrome, chylothorax, and respiratory failure requiring ventilation occurred infrequently in both cohorts; however, atelectasis or secretion retention requiring bronchoscopy occurred less often in the VATS group. The prevalence of all other complications was similar for the 2 cohorts. Significantly fewer patients in the VATS group $(18 / 66,27.3 \%)$ experienced at least 1 complication compared with the open group $(327 / 686,47.8 \% ; P=.005)$.

\section{DISCUSSION}

VATS is widely accepted for certain indications, but the use of this technique to perform anatomic lung resection for lung cancer remains controversial. The potential advantages of VATS lobectomy include a smaller incision with better postoperative mechanics and less postoperative pain, a lower incidence of postoperative atrial arrhythmias, fewer respiratory complications, earlier chest tube removal, and a shorter length of hospital stay, among others. Supporters believe that VATS lobectomy can be performed while preserving the oncologic principles of open lobectomy in terms of the ability to achieve a complete (R0) resection and per-
TABLE 2. Perioperative outcomes

\begin{tabular}{lllr}
\hline & VATS $(\mathbf{n}=66)$ & Open $(\mathbf{n}=\mathbf{6 8 6})$ & $\boldsymbol{P}^{*}$ \\
\hline Operative time, min & $117.5 ; 61-450$ & $171.5 ; 40-425$ & $<.001$ \\
$\begin{array}{l}\text { Lymph node stations } \\
\quad 7(5-11)\end{array}$ & $7(3-13)$ & .418 \\
$\quad$ sampled $\dagger$ & & & \\
Lymph nodes retrieved $\dagger$ & $15 ; 5-48$ & $19 ; 2-83$ & .147 \\
R1/R2 resection, $\mathrm{n}(\%)$ & $0(0)$ & $16(2.3)$ & .368 \\
Chest tube drainage, $\mathrm{mL} \ddagger$ & $987 ; 140-3382$ & $1504.5 ; 8-25,139$ & .001 \\
Hospital length of stay, $\mathrm{n}(\%) \S$ & $4.5 ; 1-19$ & $7 ; 0-99$ & $<.001$ \\
Operative mortality, $\mathrm{n}(\%)$ & $0(0)$ & $11(1.6)$ & 1.0 \\
\hline
\end{tabular}

VATS, Video-assisted thoracoscopy. Values are median; range unless specified by $n$ $(\%)$. *Conditional logistic regression for categoric variables (exact tests when appropriate) and repeated-measure analysis of variance for continuous variables accounting for the propensity score stratification. $\dagger$ Lymph node dissection cases only $(29$ in the VATS group and 353 in the OPEN group); data were missing for 1 subject in the thoracotomy group for lymph nodes retrieved. $\ddagger$ Data were missing for 12 subjects in the thoracotomy group. §Data were missing for 2 subjects in the thoracotomy group.

form an adequate lymph node dissection. Similar overall 5 -year survival was noted in a randomized trial comparing VATS with open lobectomy. ${ }^{2}$ Critics would point out that another randomized trial demonstrated no clear advantages to the use of VATS lobectomy because patients experienced a similar length of stay and perioperative morbidity ${ }^{1}$ and that the majority of data supporting VATS lobectomy have been in the form of retrospective case series, with only a few large case-control series reported. ${ }^{7}$

We examined the database of the ACOSOG Z0030 clinical trial to identify patients who underwent anatomic lung resection (lobectomy, bilobectomy, or segmentectomy) through a VATS or open approach with the goal of performing a case-control study that would compare VATS lobectomy with open lobectomy. There are several advantages to using the ACOSOG Z0030 data set, including the fact that this was a large (1023 patients) prospective, multicenter, phase III clinical trial. ${ }^{11}$ Strict eligibility criteria for enrollment were used, and patients underwent a standardized preoperative clinical and imaging evaluation with computed tomography scans performed within 60 days of surgery. Operative reports were reviewed by 1 of 2 main investigators. Data collection was prospective, uniform, and of high quality. Collection of data regarding outcomes and complications was standardized as to the definitions and types of variables collected. However, this was a secondary analysis of data from a randomized trial originally designed to compare the results of lymph node sampling with those of lymph node dissection in patients with resectable lung cancer. Not unexpectedly, we observed important baseline differences in the VATS lobectomy group compared with the open lobectomy group (Table 1). The patients in the VATS group were older, were more likely to have non-squamous histology, and had a better Eastern Cooperative Oncology Group performance status; in addition, most were operated on by 1 surgeon. 
TABLE 3. List of complications

\begin{tabular}{|c|c|c|c|c|c|}
\hline Variable & VATS $(n=66)$ & Open* $(n=686)$ & $\boldsymbol{P} \dagger$ & OR & $\mathbf{9 5} \% \mathbf{C I}$ \\
\hline Air leak $>7 \mathrm{~d}$ & $1(1.5)$ & $50(7.3)$ & 0.23 & $0.01-1.46$ & .155 \\
\hline Chest tube drainage $>7 \mathrm{~d}$ & $1(1.5)$ & $74(10.8)$ & 0.15 & $0.004-0.9$ & .029 \\
\hline Chylothorax, stopped spontaneously & $0(0)$ & $1(0.2)$ & & & 1.0 \\
\hline Chylothorax, required reoperation & $0(0)$ & $6(0.9)$ & & & 1.0 \\
\hline Hemorrhage, required transfusion & $2(3.0)$ & $13(1.9)$ & 1.17 & $0.12-6.03$ & .69 \\
\hline Hemorrhage, required reoperation & $1(1.5)$ & $9(1.3)$ & 1.02 & $0.02-8.32$ & 1.0 \\
\hline Atelectasis or secretional retention, requiring bronchoscopy & $0(0)$ & $43(6.3)$ & & & .035 \\
\hline Recurrent nerve palsy & $0(0)$ & $3(0.4)$ & & & \\
\hline Phrenic nerve palsy & $0(0)$ & $3(0.4)$ & & & 1.0 \\
\hline Atrial arrhythmia & $6(9.1)$ & $88(12.9)$ & 0.53 & $0.18-1.31$ & .185 \\
\hline Ventricular arrhythmia & $0(0)$ & $8(1.2)$ & & & .587 \\
\hline Respiratory failure, requiring ventilation & $0(0)$ & $5(3.7)$ & & & .381 \\
\hline MI & $1(1.5)$ & $6(0.9)$ & 1.56 & $0.03-16.0$ & .538 \\
\hline CVA & $0(0)$ & $3(0.4)$ & & & 1.0 \\
\hline DVT & $0(0)$ & $5(0.7)$ & & & 1.0 \\
\hline BP fistula & $0(0)$ & $1(0.2)$ & & & 1.0 \\
\hline Empyema & $1(1.5)$ & $3(0.4)$ & & & .473 \\
\hline Wound infection & $0(0)$ & $3(0.4)$ & & & 1.0 \\
\hline Sepsis & $0(0)$ & $3(0.4)$ & & & 1.0 \\
\hline ARDS & $0(0)$ & $2(0.3)$ & & & 1.0 \\
\hline Pulmonary embolus & $0(0)$ & $4(0.6)$ & & & 1.0 \\
\hline Other postoperative outcome & $12(18.2)$ & $195(28.5)$ & 0.63 & $0.30-1.26$ & .218 \\
\hline At least 1 complication & $18(27.3)$ & $327(47.8)$ & 0.44 & $0.23-0.81$ & .005 \\
\hline
\end{tabular}

ARDS, Adult respiratory disease syndrome; $B P$, bronchopleural; $C I$, confidence interval; $C V A$, cerebrovascular accident; $D V T$, deep vein thrombosis; $M I$, myocardial infarction; $O R$, odds ratio; VATS, video-assisted thoracoscopy. OR and CI are presented for outcomes with $\geq 1 \%$ in each group. *Data were unavailable for 2 subjects in the open lobectomy group. $\dagger$ Conditional logistic regression accounting for the propensity score stratification. Exact tests were appropriate.

To deal with the biases inherent in any nonrandomized comparison, we analyzed the data using the technique of propensity scores, a method of case-matching. Propensity scores are a class of multivariate statistical methods that identify groups of patients who have similar chances of receiving one treatment or another from within a given study population. Using propensity scores avoids some of the problems associated with case matching by creating a model where the differences between the groups are "compressed" into a single score. ${ }^{12}$ In this analysis, age, gender, histology, performance status, tumor location, and clinical T stage (T1 vs T2) were used to build a propensity score for choice of treatment. Significant differences in outcomes between treatment groups can then be attributed to the difference in treatments as long as the patients in the different treatment groups have similar propensity scores. However, we were not able to include the variable of operating surgeon in our propensity score model, and therefore this remains an unresolved potential source of confounding in this analysis. A total of 9 surgeons performed VATS lung resections in the ACOSOG Z0030 trial. However, a single surgeon performed most of the VATS procedures. This means that one cannot exclude the possibility that the results achieved in the VATS group were due to the surgeon and hospital perform- ing the VATS procedures and not just to the fact that the patient underwent a VATS procedure.

The main finding of this secondary analysis of data from the ACOSOG Z0030 trial is that, after matching for preoperative variables such as age, gender, performance status, histology and T stage, patients who underwent VATS lobectomy had a statistically significant shorter median length of stay compared with the open group. A decrease in postoperative length of stay after VATS lobectomy has been reported by many but not all studies comparing clinical outcomes of VATS lobectomy and open thoracotomy and lobectomy. ${ }^{6,7}$ This has been variously attributed to decreased postoperative pain or earlier removal of chest tubes in patients undergoing VATS lobectomy. In the current study, we observed fewer instances of chest tube drainage lasting greater than 7 days in the VATS group compared with the open group despite similar occurrences of air leak lasting greater than 7 days. Median total chest tube drainage was also significantly less for the VATS group compared with the open group. Data regarding postoperative pain were not collected in patients enrolled in the Z0030 trial; therefore, we cannot comment on the likelihood that decreased pain contributed to the shorter postoperative length of stay observed in the VATS group. In addition, as others have pointed out, chest tube 
management may differ between surgeons and institutions performing and not performing VATS lobectomy. ${ }^{6}$ Parameters for chest tube management were not defined in the ACOSOG Z0030 trial; therefore, the effect of institutional and surgeon differences in chest tube management cannot be excluded as an explanation for the observations that were identified in this analysis.

Another proposed benefit of VATS lobectomy has been a decrease in postoperative complications. In the current analysis, we observed that the median number of patients experiencing at least 1 complication was lower in the VATS group. This is similar to the report of Park and colleagues, ${ }^{8}$ who matched 122 patients undergoing VATS lobectomy with 122 patients undergoing open lobectomy and observed fewer overall complications in the VATS group. In addition to the decreased likelihood of chest tube drainage lasting more than 7 days for the VATS group that was mentioned above, we observed that patients in the VATS cohort underwent fewer postoperative bronchoscopies for atelectasis or secretion retention. This finding may be related to the combination of decreased pain, inflammation, and better mobilization of secretions because of improved postoperative chest wall function in the VATS lobectomy group. A decrease in postoperative respiratory complications after VATS lobectomy compared with open lobectomy has been shown in some studies, especially in the elderly, ${ }^{9}$ but not in other analyses. ${ }^{6,7}$

We noted a similar prevalence of atrial fibrillation in both the VATS and open lobectomy groups, a finding that others have also reported. ${ }^{8}$ This would suggest that the mechanical effects of dividing pulmonary vessels, denervation, or other neurohumoral factors related to lung resection per se rather than effects related to the size of the incision were responsible for postoperative arrhythmias. The recent meta-analysis by Yan and colleagues ${ }^{7}$ found that the occurrence of postoperative arrhythmias (based on an analysis of 5 studies) in VATS lobectomy cases compared with open lobectomy cases was similar (relative risk [RR], 0.99; 95\% confidence interval $[\mathrm{CI}], 0.52-1.91 ; P=.98)$. The current analysis found that the occurrence of other cardiovascular complications (myocardial infarction, stroke, pulmonary embolus, or ventricular arrhythmias) was similar for the 2 groups. Finally, the number of instances of bleeding requiring transfusion or reoperation was also similar for the 2 cohorts.

Survival data are pending for the ACOSOG Z0030 study participants, so the oncologic efficacy of VATS and open lobectomy cannot be determined from the current analysis. However, the median number of R1 or R2 resections was similar for the 2 groups, supporting the concept that VATS lobectomy is an oncologically sound operation. Critics of the VATS approach have raised concerns about the adequacy of lymph node dissection compared with open lobectomy. We found that in those patients randomized to lymph node dissection, the number of lymph node stations sampled and the total number of lymph nodes removed were similar for both VATS lobectomy and open lobectomy. Kirby and colleagues, ${ }^{1}$ in a randomized trial, noted no difference in the total number of lymph nodes sampled in VATS lobectomy compared with muscle-sparing thoracotomy and lobectomy (total number of lymph nodes: VATS $9.5 \pm 3.6$ vs muscle-sparing thoracotomy $9.3 \pm 4.3$ ). In 2002, Sagawa and colleagues ${ }^{15}$ performed VATS lobectomy and lymph node dissection followed by immediate thoracotomy to look for "remnant" lymph nodes in 29 patients. They found an average of 1.2 remnant nodes of an average total of 40.3 lymph nodes for right-sided cases and 1.2 remnant nodes of an average total of 37.1 nodes for left-sided cases. Watanabe and colleagues ${ }^{16}$ examined lymph nodes from 350 patients undergoing lobectomy with systematic lymph node dissection (191 VATS cases and 159 open lobectomy cases) and found that the total number of mediastinal lymph nodes dissected was similar for each group. A recent meta-analysis of 21 studies ( 2 randomized and 19 nonrandomized) performed by Yan and colleagues ${ }^{7}$ found similar local recurrence rates for VATS lobectomy compared with open lobectomy (RR, 0.64; 95\% CI, 0.35-1.35; $P=.24$ ). Their analysis suggested that VATS lobectomy for lung cancer was associated with a reduced systemic recurrence rate (RR, $0.57 ; 95 \%$ CI, $0.3-0.95 ; P=.03$ ) and improved 5-year all-cause mortality rate for VATS (RR, 0.66 ; $95 \% \mathrm{CI}, 0.45-0.97$; $P=.04)$.

\section{CONCLUSIONS}

This secondary analysis of data from ACOSOG Z0030 suggests that VATS lobectomy is an appropriate procedure for the treatment of early-stage lung cancer in terms of mortality and complications when compared with open lobectomy. VATS lobectomy may offer advantages in terms of decreased length of stay and overall fewer complications. However, the results of this analysis should be interpreted carefully. Although we used propensity scores to adjust for important baseline differences, such as age, gender, performance status, histology, and T1 or T2 stage between the VATS lobectomy and open lobectomy groups, we were not able to include the variable of operating surgeon in our propensity score model. This remains an unresolved potential source of confounding in this analysis. The outcomes observed for VATS lobectomy are inextricably linked with the surgeon and hospital center that enrolled most of those patients into the ACOSOG Z0030 trial. However, the current authors believe that we have done all that we can to make the comparisons between the VATS and open lobectomy cohorts as fair as possible. Long-term follow-up data are needed to judge the oncologic equivalence of the 2 approaches. The mature results of ACOSOG Z0030 should provide more information regarding local recurrence and overall survival of those patients undergoing VATS lobectomy versus open lobectomy. 
The authors thank the investigators, site research teams, and patients who participated in this study, and Ken Ross for his assistance with manuscript preparation.

\section{References}

1. Kirby TJ, Mack MJ, Landreneau RJ, et al. Lobectomy-video-assisted thoracic surgery versus muscle-sparing thoracotomy: a randomized trial. J Thorac Cardiovasc Surg. 1995;109:997-1002.

2. Sugi K, Kaneda Y, Esato K. Video-assisted thoracoscopic lobectomy achieves a satisfactory long-term prognosis in patients with clinical stage IA lung cancer. World J Surg. 2000;24:27-31.

3. Craig SR, Leaver HA, Yap PL, Pugh GC, Walker WS. Acute phase responses following minimal access and conventional thoracic surgery. Eur J Cardiothorac Surg. 2001;20:455-63

4. Shigemura N, Akashi A, Nakagiri T, Ohta M, Matsuda H. Complete vs. assisted thoracoscopic approach: a prospective randomized trial comparing a variety of video-assisted thoracoscopic lobectomy techniques. Surg Endosc. 2004;18:1492-7.

5. Swanson SJ, Herndon JE 2nd, D'Amico TA, et al. Video-assisted thoracic surgery lobectomy: report of CALGB 39802 — a prospective, multi-institution feasibility study. J Clin Oncol. 2007;25:4993-7.

6. Whitson BA, Groth SS, Duval SJ, Swanson SJ, Maddaus MA. Surgery for earlystage non-small cell lung cancer: a systematic review of the video-assisted thoracoscopic surgery versus thoracotomy approaches to lobectomy. Ann Thorac Surg. 2008;86:2008-18.

7. Yan TD, Black D, Bannon PG, McCaughan BC. Systematic review and metaanalysis of randomized and non-randomized trials on safety and efficacy of video-assisted thoracic surgery lobectomy for early-stage non-small-call lung cancer. J Clin Oncol. 2009;27:2553-62.

8. Park BJ, Zhang H, Rusch VW, Amar D. Video-assisted thoracic surgery does not reduce the incidence of postoperative atrial fibrillation after pulmonary lobectomy. J Thorac Cardiovasc Surg. 2007;133:775-9.

9. Cattaneo SM, Park BJ, Wilton AS, et al. Use of video-assisted thoracic surgery for lobectomy in the elderly results in fewer complications. Ann Thorac Surg. 2008; 85:231-6.

10. Mckenna RJ, Houch W, Fuller CB. Video-assisted thoracic surgery lobectomy: experience with 1,100 cases. Ann Thorac Surg. 2006;81:421-6.

11. Allen MS, Darling GE, Pechet TTV, et al. Morbidity and mortality of major pulmonary resections in patients with early-stage lung cancer: Initial results of the randomized, prospective ACOSOG Z0030 Trial. Ann Thorac Surg. 2006;81: 1013-20.

12. Blackstone EH. Comparing apples and oranges. J Thorac Cardiovasc Surg. 2002; 123:8-15.

13. Rosenbaum PR, Rubin DB. The central role of the propensity score in observational studies for causal effects. Biometrika. 1983;70:41-55.

14. D'Agostino RB. Propensity score methods for bias reduction in the comparison of a treatment to a non-randomized control group. Stat Med. 1998;17:2265-81.

15. Sagawa M, Sato M, Sakurada A, et al. A prospective trial of systematic nodal dissection for lung cancer by video-assisted thoracic surgery: can it be perfect? Ann Thorac Surg. 2002;73:900-4.

16. Watanabe A, Koyanagi T, Ohsawa H, et al. Systematic node dissection by VATS is not inferior to that through and open thoracotomy: a comparative clinicopathologic retrospective study. Surgery. 2005;138:510-7.

\section{Discussion}

Dr John D. Mitchell, MD (Denver, Colo). The ACOSOG Z30 study was a randomized multi-institutional prospective trial designed to assess the relative merits of mediastinal lymph node dissection versus sampling in patients with early-stage lung cancer, both in terms of the perioperative morbidity of the 2 procedures and the long-term oncologic outcomes. The first has been reported, as Walter said, and we await the data regarding recurrence and survival.

This current study examines a subset of the Z30 patients who underwent a lobectomy, bilobectomy, or segmentectomy. Through the magic of propensity scoring, these patients have been distilled down into 2 matched groups: 66 patients who underwent anatomic lung resection via a thoracoscopic approach and 686 patients who had an open procedure. The VATS group had shorter operative times, less chest tube drainage, shorter chest tube duration, shorter hospitals stays, less respiratory problems requiring intervention, and less complications overall. I was gratified to see that your findings matched my own personal observations about VATS procedures, but I have a couple questions for you.

First, the majority of the VATS procedures, $82 \%$, were done by 1 surgeon. How do we really know that the differences that you are reporting today are related to the 2 different surgical approaches? An alternative possibility is that these results just reflect practice differences between the surgeon and the rest of the ACOSOG participants. For example, if the VATS surgeon in question is more liberal with chest tube removal, with less regard for the output, the chest tube duration is going to be shorter, and the hospital stay will probably be shorter.

Dr Scott. That is the key confounder in this analysis, as I mentioned. The protocol did not mandate how chest tubes were managed. Therefore, a surgeon's practice pattern, for example, his/her willingness to remove a chest tube that drained $400 \mathrm{~mL}$ in the previous 24 hours compared with waiting for drainage to decrease to $250 \mathrm{~mL}$ in the previous 24 hours, would make a difference in the timing of chest tube removal. End points such as chest tube removal have to be looked at with that in mind. However, regarding other end points such as mortality, lymph node dissection, and so on, I believe the study shows that VATS lobectomy can be done and achieve similar results to open lobectomy. Those end points are perhaps less affected by the fact that 1 surgeon did most of the procedures in the VATS group. The study demonstrates that VATS lobectomy can achieve those similar goals.

Dr Mitchell. Second, there were 10 times as many patients in the open resection group compared with the VATS group. There were other differences as well, such as in the performance status. Do you have any concerns about the validity of your results based on these discrepancies between the 2 groups given the statistical methods and scoring variables used?

Dr Scott. I think propensity matching is probably one of the most sophisticated methods we have for performing a nonrandomized comparison such as we did here. The alternative is to do something called case matching where you find 66 patients in the open resection group who are similar to the 66 patients in the VATS lobectomy group. Problems with the case-matching approach include accurately identifying similar patients from each group (ie, how does one determine how similar is similar enough?) and the fact that a significant number of patients would be excluded from the analysis. This can actually increase the amount of bias in the analysis. The fact that one has a large control group is actually favorable for this type of statistical analysis.

Dr Mitchell. Finally, a VATS approach was used selectively in the Z30 trial at the discretion of the operating surgeon. Could you comment on how this fact might have affected the results in your trial?

Dr Scott. I hope that the differences we saw in baseline characteristics were corrected by the propensity matching, and therefore the patients were as similar as possible. That gets at the heart of doing this kind of nonrandomized comparison. The choice by a given surgeon of whether to offer a VATS or open procedure should be accounted for by the statistical method because both groups of patients should be similar. 
Dr Robert Cerfolio, MD (Birmingham, Ala). Walter, this is an interesting article. However, I come to meeting after meeting and listen, and I keep hearing that VATS is better, and I keep waiting for some data to convince me, and, once again, no offense, but this is not it.

Did you compare Dr McKenna's open cases with Dr McKenna's VATS cases? Because that is really what the article was. In other words, did you look at the time frame for Rob's open thoracotomies and were the chest tubes removed as quickly as in the VATS cases? I know we do at UAB, and we do some, although not many VATS lobectomies. To me, that would be the only important data and analysis from this study.

Finally, because I continue to hear this meeting after meeting, I am performing VATS lobectomy now, even some robotic work, and I see no difference in chest tube management. Perhaps I am just a much worse VATS surgeon than you guys or maybe you guys just don't do your thoracotomies the way I do: spare the rib, most of the muscle, and all the intercostal nerves. Maybe you do not fast track these patients or have the mindset that their tubes can come out after 1 or 2 days and they can go home after 3 days and return to full activities after 3 or 4 weeks. I think if you just treated the open cases more like the VATS cases you might not see a difference. Can you comment on that?

Dr Scott. Those are 2 interesting points. I don't think these data settle the question, and perhaps a randomized trial is necessary, but I am not sure there is equipoise in the general thoracic surgery community to do such a trial.

Dr McKenna did not have many patients who underwent the open procedure to compare; therefore, the type 2 error would probably be great, so we could not do that. We did perform an exploratory analysis looking at centers that performed VATS lobectomy compared with centers that performed open lobectomy, and there was a trend for a shorter length of stay with open cases as well in centers that performed VATS lobectomy as opposed to those centers that performed only open resections. Some people might see that as a criticism therefore or a reason to think that VATS is not as good; I would argue the opposite, that the experience with VATS lobectomy led the surgeons to fast track their open resections, if you will, and that there is something about the experience of doing VATS lobectomies that benefits all the patients.

Dr Cerfolio. Okay, my only point is I would just be careful with your conclusions given the methodology of this study.

Dr David Follette, MD (Sacramento, Calif). Dr Scott, this was a superbly presented article. I do, however, share Dr Cerfolio's concerns. We now know that VATS lobectomy can be done safely by experienced VATS surgeons. We also know there may be some short-term advantages. It is not known if these apparent advantages will really make any long-term difference.

I too share concerns about your data where the majority of cases were done by a single surgeon who is recognized as the world's authority and has the largest experience. We are all aware of Dr McKenna's published results, and they certainly set a lofty standard for all surgeons who choose this approach. If there were a broadbased series, would the results for VATS lobectomy be comparable?

Thus, I have grave concerns that essentially a single-surgeon series be compared with a group of other surgeons doing open lobec- tomies. I don't believe your subset analysis is statistically valid. Could you please comment on this?

Dr Scott. There are always concerns when one does post hoc or subset analyses such as this. Basically I would say that the data are what they are. They have been analyzed in an excellent fashion by the ACOSOG statisticians, and these are the conclusions we can come to. As I mentioned, they need to be interpreted in light of the fact that 1 surgeon did most of the VATS procedures.

Dr Follette. Don't you think this is a lot like all the published and presented studies on off-pump versus on-pump coronary bypass surgery? Do you believe that we should answer the question as to open versus VATS lobectomy in a similar manner that answered the off versus on-pump surgery question? As you may recall, Emory University conducted a statistically relevant study that randomly assigned patients to either technique. All procedures were done by a single cardiac surgeon with extensive experience with both techniques. As you may recall, when the data were analyzed, there was no statistical difference in outcomes or complications between these 2 techniques. Do you think that if a highvolume center, with extensive experience with both techniques, would be willing to do such a study that this would answer the question once and for all?

Dr Scott. I think that would be a good idea, and I leave it up to those centers to take on that task.

Dr Douglas Wood, MD (Seattle, Wash). Walter, nicely presented. But, I have to take the previous criticisms and perhaps emphasize them even greater.

It's not legitimate to title the article the way it is titled. This is not really a comparison of VATS versus open. This is a comparison of Rob McKenna versus the rest of the thoracic surgeons in the United States. I mean you could title the article as that, "Preoperative Outcomes of Resection for Lung Cancer: A Comparison of Dr McKenna and the Rest of the US Thoracic Surgeons.' It would be an equally legitimate title and a data analysis, albeit not as interesting or provocative as a title comparing VATS with open. I question whether this is really something that we should put in the literature because of that. This problem of confounding, of Rob McKenna doing $82 \%$ of the VATS cases, it just can't be overcome statistically.

I challenge your statement that there is not equipoise in the thoracic surgery community to achieve this as a randomized trial. Dr Mitchell and I can have completely different opinions about the validity of VATS lobectomy. He can think that VATS is absolutely better, and I can be cynical and not sure about that, just like Dr Cerfolio. That is equipoise in the thoracic surgery community. Maybe we don't have it individually, but we don't have to as long as I respect John Mitchell's point of view and he respects my point of view; we actually have the capability of conducting a randomized trial because we recognize that it is an unanswered question. I request that we as thoracic surgeons get off of our position that this has already been defined, because it has not, and through ACOSOG or other sources put forward a randomized trial and work together.

Dr Scott. In response to your initial comments, I would point out the strengths of this study: the standardized definitions of complications and outcomes, the prospective data collection, and the carefully audited data (unlike many national databases). Useful conclusions can be drawn from these data. 
Regarding your second point, at meetings such as the general thoracic surgery club, whenever someone asks for a show of hands of those willing to participate in a randomized study of VATS versus open lobectomy, nobody raises his or her hand. That demonstrates a lack of equipoise in the thoracic surgery community in that you may think open is better, I may think VATS is better, and I'm not willing to randomize my patients. Unless these attitudes change, there does not currently seem to be much likelihood of a randomized trial succeeding. 